\title{
Wheat Genotypes Evaluated under North Eastern Plains Zone of the Country for Genotype X Environment Interaction Analysis
}

\author{
Ajay Verma* and G. P. Singh \\ ICAR-Indian Institute of Wheat \& Barley Research, Karnal 132001 Haryana, India \\ *Corresponding author
}

\section{A B S T R A C T}

\begin{abstract}
AMMI analysis observed highly significant values of environments, GxE interaction effects and genotypes effects for both the years of study. Lower values of EV1 during (2016-17) ranked genotypes as (G6, G2, G8); D1 for (G6, G2, G8); values of ASTAB1 for (G6, G2, G8) while SIPC1 for (G7, G1, G5) genotypes. EV2 measure pointed towards (G6, G2, G9) as desirable, D2 for (G6, G2, G8), whereas as per SIPC2 were (G5, G7, G8) \& ASTAB2 opted for (G6, G2, GxE interaction. Ranked values of EV3 preferred G2, G6, G8; SIPC3 pointed towards G5, G8, G1; D3 identified G2, G6, G8 and ASTAB3 considered G2, G6, G8 wheat genotypes. Numerical values of D7 ranked G6, G2, G8; SIPC7 chosen G8, G1, G5. EV7 pointed towards G2, G6, G8 \& ASTAB7 identified G9, G1, G4 as desirable over the studied environments. Composite measure MASV selected G6, G2, G9 \& MASV1 cited as G6, G2, G8 would be desirable. Genotypes G8, G2 and G6 by Mean, GAI, HM, PRVG and MHPRVG measures would be of choice across environments of study. Biplot analysis of studied measures exhibited three major clusters and most of the measures clubbed in first cluster. Seven significant IPCA's were used to calculate AMMI based measures during second year (2017-18) as accounted more than $96.6 \%$. Minimum values of EV1 ranked (G9, G6, G1), D1 pointed (G9, G6, G1), ASTAB1 for (G9, G6, G1) and for SIPC1 were (G7, G5, G10). EV2 pointed towards (G9, G1, G6) as desirable, for values of D2 genotypes were (G9, G1, G6) as per criterion of SIPC2 were (G7, G5, G10) \& ASTAB2 favoured (G9, G1, G6). ASV and ASV1 recommended (G9, G1, G6) as of stable performance. D7 expressed minimum values G9, G1, G6; SIPC7 observed G5, G9, G10, measure EV7 pointed towards G1, G5, G4. ASTAB7 identified G9, G1, G4 as desirable. Composite measure MASV selected G9, G5, G1 and MASV1 as G9, G1, G5 for desirable performance. Genotypes G8 G9 by Mean, G7, G4 by GAI \& HM, G4, G10 by PRVG and G7, G4 by MHPRVG measures based on yield of genotypes across environments of study. Association analysis among AMMI measures and yield based analytic measures by multivariate hierarchical Ward's clustering approach grouped into four major clusters. Largest group I clubbed measures as MASV1, ASV, MASV, D2, D3, D7, with EV2, EV3, SIPC5, SIPC7, EV7. Group II contains ASTAB1, ASTAB2, ASTAB5, D1, D5, EV1, EV5, ASV1 whereas yield based measures exhibited close proximity and placed close to each other as in separate group.
\end{abstract} \begin{tabular}{l|lll} 
Keywords & G9). ASV and ASV1 recommended (G6, G2, G9) genotypes possessing stable performance as measures used $56.9 \%$ of
\end{tabular}

\section{Introduction}

Quite large number of statistical methods are available for analysis of multi environment trials, aimed to subdivide the complex GxE interaction into simpler and more meaningful responses of genotypes among studied environments (Agahi et al., 2020). Multi Environment trials of cereal crops had been planned to have efficient estimation of main and interaction effects (Bocianowski et al., 2019). The procedures vary from univariate parametric, non-parametric and multivariate models. AMMI model ((Additive main effects and multiplicative interaction), incorporates both additive and multiplicative components with aim to summarize the genotypeenvironment interaction (Guilly et al., 2017). 
Although, analysis of variance (ANOVA) provided an interaction sum of squares that would be difficult to interpret and the prediction of yields in different environments is not easy (Kamila et al., 2016). AMMI separates the additive variance from the multiplicative variance and then applies principal component analysis (PCA) to the interaction portion to a new set of coordinate axes that explains in more detail the interaction pattern (Tekdal \& Kendal, 2018).

AMMI analysis has been shown to be effective because it captures a large portion of the GxE sum of squares, clearly separating main and interaction effects that provide researchers with different opportunities (Gauch 2013; Tena et al., 2019). Prime objectives of this study are (i) AMMI based measures depend on utilization of significant principal components (ii) explore the association among AMMI along with yield and adaptability measures.

\section{Materials and Methods}

North Eastern Plains Zone of India comprises eastern Uttar Pradesh, Bihar, Jharkhand, Assam and plains of West Bengal. Nine advanced wheat genotypes twelve locations and eleven genotypes at fifteen locations were evaluated under field trials at of north eastern plains zone during 2017-18 and 2018-19 cropping seasons respectively. Field trials were conducted at research centers in randomized complete block designs with three replications.

Recommended agronomic practices were followed to harvest good yield. Details of genotype parentage along with environmental conditions were reflected in tables $1 \& 2$ for ready reference. AMMI first calculate genotype and environment additive effect using analysis of variance (ANOVA) and then analyse residual from these model using principal components analysis (PCA). AMMI stability value (ASV) was initially proposed by Purchase (1997) to quantify the stability measure by considering relative weight of $\mathrm{IPCA}_{1}$ and IPCA 2 scores. In certain cases where more than two IPCAs were significant, ASV failed to encompass all the variability explained by GEI. In order to overcome this difficulty, Zali et al., (2012) attempted to present a modified version ASV i.e., Modified ASV which would cover all available IPCAs.

But in doing so, Zali et al., (2012) interpreted the formula of ASV incorrectly compared to the original formula of Purchase (1997). In the present study the MASV of Zali et al., (2012) and a revised version of MASV (Ajay et al., 2019) were compared with other AMMI based measures. The description of widely used measures based on AMMI analysis was mentioned for completeness.

\begin{tabular}{|c|c|c|c|c|}
\hline Zobel & 1994 & EV1 & EVF & $\mathrm{EV}=\sum_{n=1}^{N} \lambda_{\mathrm{in}}^{2} / n$ \\
\hline Sneller et al., & 1997 & SIPC1 & SIPCF & $\operatorname{SIPC}=\sum_{n=1}^{M} \lambda_{n}^{0.5} \gamma_{m}$ \\
\hline Purchase & 1997 & ASV & & $\mathrm{ASV}=\left[\left(\frac{\operatorname{SSIPC} 1}{\operatorname{SSIPC} 2} * \mathrm{PCI}\right)^{2}+(\mathrm{PC} 2)^{2}\right]^{1 / 2}$ \\
\hline Annicchiarico & 1997 & D & & $\mathrm{D}=\sqrt{\sum_{n=1}^{Y}\left(f_{n n} \gamma_{m}\right)^{2}}$ \\
\hline $\begin{array}{l}\text { Rao and } \\
\text { Prabhakaran }\end{array}$ & 2005 & ASTAB & & $A S T A B=\sum_{n=1}^{n} \lambda_{n} \gamma_{n i}^{2}$ \\
\hline
\end{tabular}




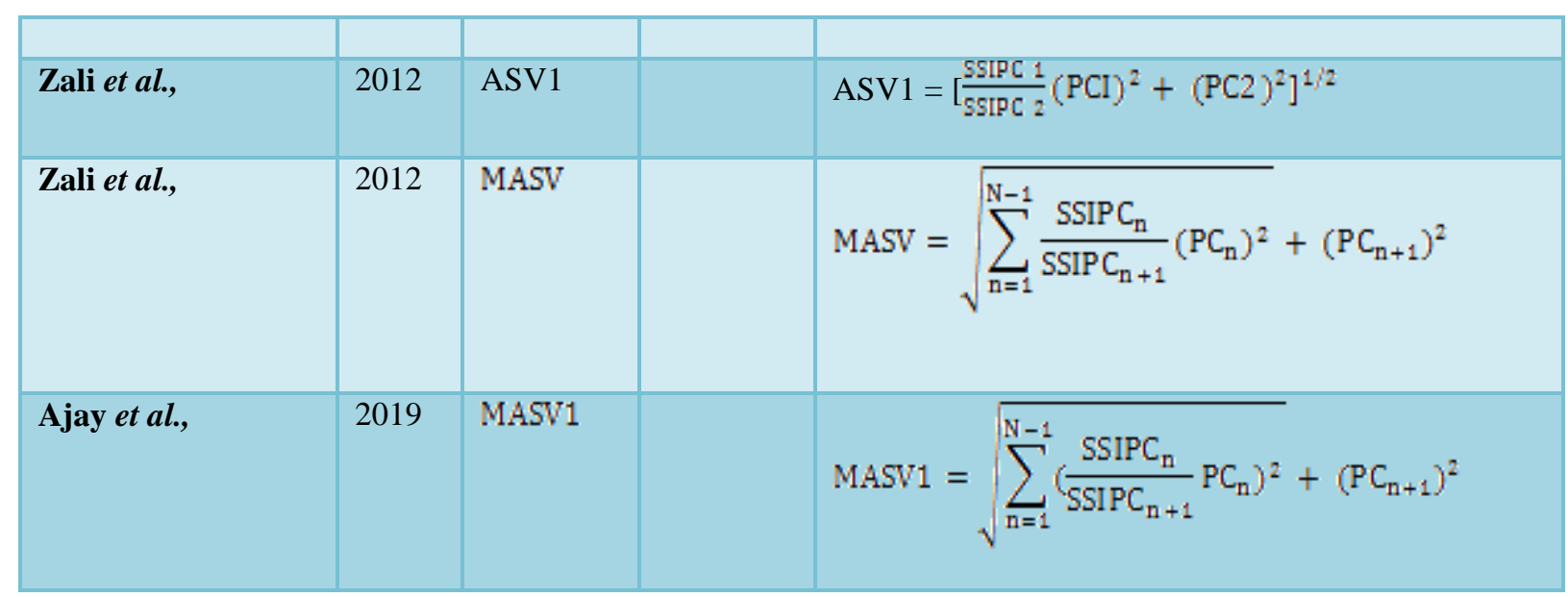

AMMI analysis was performed using AMMISOFT version 1.0, available at https://scs.cals.cornell.edu/people/ hughgauch/ and SAS software version 9.3. AMMI based measures were compared with recent analytic measures of adaptability calculated as the relative performance of genetic values (PRVG) and MHVG (Harmonic mean of Genetic Values), based on the harmonic mean of the genotypic values across different environments.

Another harmonic mean based measure of the relative performance of the genotypic values (MHPRVG) for the simultaneous analysis of stability, adaptability and yield (Resende \& Durate, 2007).

$\mathrm{PRVG}_{\mathrm{ij}}=\mathrm{VG}_{\mathrm{ij}} / \mathrm{VG}_{\mathrm{i}}$

$\mathrm{MHVG}_{\mathrm{i}}=$ Number of environments $/ \sum_{i=1}^{k} \frac{1}{X_{i}}$ MHPRVG $_{\text {i. }}$

$=$ Number of environments $/ \sum_{j=1}^{k} \frac{1}{p R V G_{i j}}$

$\mathrm{VG}_{\mathrm{ij}}$ is the genotypic value of the $\mathrm{i}$ genotype, in the $j$ environment, expressed as a proportion of the average in this environment. Geometric adaptability index (GAI) (Mohammadi \& Amri, 2008) was calculated as $\sqrt[n]{\prod_{k=1}^{\mathrm{n}} \overline{\mathrm{x}}_{\mathrm{k}}}$; in which $\overline{\mathrm{x}}_{1}, \overline{\mathrm{x}}_{2,}, \overline{\mathrm{x}}_{3, \ldots} \overline{\mathrm{x}}_{\mathrm{m}}$ are the mean yields of the first, second and mth genotype across environments and $\mathrm{n}$ is number of environments. Genotypes with higher values of GAI are desirable

\section{Results and Discussion}

AMMI analysis provided a better understanding of the GxE interaction through analysis of variance, facilitated discriminating environments and adaptability of the genotypes to specific environments. Actually AMMI fits a family of models with retaining $0,1,2$, or more significant interaction principal components (IPCs).

\section{First year (2017-18)}

\section{AMMI analysis}

Model diagnosis is required to determine the suitable AMMI model for a given dataset while satisfying statistical and practical considerations. FR-tests at the 0.01 level diagnose AMMI6. Sums of squares for GxE signal and noise were $85.47 \%$ and $14.53 \%$ respectively of total GxE sum of squares. Sum of Squares for GxE signal is 3.53 times of genotypes main effects depicts narrow adaptations are important for this dataset.

Even just IPC1 alone is 1.56 times the genotypes main effects. Also note that GxE noise is 0.60 times the genotypes main 
effects. Highly significant environments, GxE interactions and genotypes effects were depicted in table 3. Large magnitude of GxE interactions for yield found in this investigation are similar to those found in other crops (Nowosad et al., 2018). AMMI derived measures based on the use of significant IPCA's were calculated as EV1, ASTAB1, SIPC1, D1 measures (only first significant IPCA), while ASV, EV2, ASTAB2, SIPC2, ASV1, D2 considered IPCA1 \& IPCA2, measures EV3, ASTAB3, SIPC3 and D3 used three IPCAs, measures EV5, ASTAB5, SIPC5 \& D5 (based on five IPCAs), and finally EV7, ASTAB7, SIPC7 and D7 measures utilized all significant IPCAs.

Significant GxE interactions sum of squares was further divided into seven significant interaction principal component axes (IPCAs) mentioned in table 3. Explained variation of GxE interaction accounted by each of IPCA exploited by defined measures, as type-1 AMMI based measures benefited $37.7 \%$, type-2 measures utilized $60.4 \%$, type 3 measures used up to $73.8 \%$, type 5 measures benefited up to $92.6 \%$, while type 7 measures accounted for most of variation and utilized to the extent of benefits $99.6 \%$ (Table 3 ).

This justifies the use of AMMI derived measures based on the large numbers of IPCAs results in the most usage of GxE interaction variations (Mohammadi et al., 2015; Kendal \&Tekdal, 2016).

Lower and maximum values of EV1 ranked genotypes as (G6, G2, G8) and (G7, G1) whereas for D1 were (G6, G2, G8) and (G7, G1) values of ASTAB1 for (G6, G2, G8) and $(\mathrm{G} 7, \mathrm{G} 1)$ and for SIPC1 were $(\mathrm{G} 7, \mathrm{G} 1, \mathrm{G} 5)$ \& (G3, G4), of low yield performance (Tables 5 \& 6). EV2 measure pointed towards (G6, G2, G9) as desirable and (G1, G5) vice versa, D2 for genotypes (G6, G2, G8) \& (G1, G3), whereas as per SIPC2 were $(\mathrm{G} 5, \mathrm{G} 7, \mathrm{G} 8)$ \& (G3, G4) and of ASTAB2 were (G6, G2, G9) \& (G1, G3). In recent studies, agronomic concept of stability would be more preferred instead of static concept of stability (Nowosad et al., 2016). Using first two IPCAs in stability analysis could benefits dynamic concept of stability in identification of the stable high yielder genotypes. ASV and ASV1 recommended (G6, G2, G9) genotypes possessing stable performance and unsuitable ones were G1, G7 wheat genotypes for this zone. First two IPCAs in ASV and ASV1 measures used $56.9 \%$ of GxE interaction.

The two IPCAs have different values and meanings and the ASV parameter using the Pythagoras theorem and to get estimated values between IPCA1 and IPCA2 scores to produce a balanced measure between the two IPCA scores (Purchase, 1997). ASV and ASV1 measures had used advantages of cross validation as computations are based on first two significant IPCAs.

Ranked values EV3 preferred G2, G6, G8 and unstable performance of G1, G5 while SIPC3 pointed towards G5, G8, G1 and G4, G3 D3 identified G2, G6, G8 \& G1, G7 genotypes; ASTAB3 values considered G2, G6, G8 \& G1, G7 (Table 4). Genotypes

G6, G9, G8 preferred by least values EV5 along with higher values found for G5, G1, SIPC5 values found G8, G5, G1 and G4, G3 whereas D5 considered G6, G8, G2 as suitable \& G1, G5 as unsuitable ones; ASTAB5 selected G6, G8, G2 for stable performance \& G1, G5 would be of unsuitable choice.

D7 ranked genotypes G6, G2, G8 as of stable yield while G1 and G7would be undesirable; SIPC7 observed G8, G1, G5 of choice \& G3, G2 of unstable yield (Tables 4 and 5). EV7 pointed towards G2, G6, G8 \& G5, G1 as 
suitable and unsuitable respectively. Measure ASTAB7 identified G9, G1, and G4 as desirable and G4, G3 would be unstable over the studied environments.

Composite measure MASV selected G6, G2, G9 genotypes as of stable performance and G1, G5 not recommended for cultivation due to unstable yield behavior. More over MASV1 cited as G6, G2, G8 would be desirable and G1, G5 vice versa. Genotypes G8, G2 and G6 along with G1 \& G7 by Mean, GAI, HM, PRVG and MHPRVG measures would be of choice across environments of study.

\section{Biplot analysis}

First two significant principal components were considered for biplot analysis among the considered measures to understand the association among measures if any. The relationship among these is depicted in graph as number clusters of considered measures (Ndhlela et al., 2014). First two significant principal components of considered measures accounted for variation $85.4 \%$ of total variations.

Three clusters of studied measures were observed in Figure 1. Most of the measures clubbed in first cluster as of D1, D2, D3, D5, D7, , EV1, EV2, EV3, EV5, EV7, MASV1, ASV, MASV, ASV1, SIPC1, SIPC2, SIPC3, SIPC5, SIPC7,PRVG, MHPRVG. Second cluster comprised of Mean, HA and GAI while ASTAB1, ASTAB2, ASTAB3, ASTAB5 and ASTAB7 were in separate cluster.

\section{Second year (2018-19)}

\section{AMMI analysis}

Model diagnosis based on statistical and practical considerations observed suitability of AMMI7 as also confirmed by FR-tests at the 0.01 level of significance. The sums of squares for $\mathrm{GxE}$ signal and noise were $83.31 \%$ and $16.69 \%$ of total GxE interaction sum of squares respectively.

Accordingly, this much signal suggests AMMI7 also merits consideration. Sum of squares for GxE signal is 4.13 times that for genotypes main effects.

Hence, narrow adaptations are important for this dataset. Even just IPC1 alone is 1.73 times the genotype main effects. Also note that $\mathrm{GxE}$ noise is 0.83 times the genotype main effects. Accuracy may be improved by discarding noise, as this increases repeatability helps to simplifies conclusions and accelerates progress from selection process.

First four IPCA's contributed more than $80 \%$. IPCA1 explained $34.8 \%$ of the variation affected by interaction, while IPCA2, IPCA3 and IPCA4 accounted for 22.1, 13.8 and 10\%, respectively. Explained variation of $\mathrm{GxE}$ interaction accounted by each of IPCA exploited by defined measures, as type- 1 AMMI based measures benefited $34.8 \%$, type-2 measures utilized $56.9 \%$, type 3 measures used up to $70.8 \%$, type 5 measures benefited up to $87.5 \%$, while type 7 measures accounted for most of variation and utilized to the extent of benefits $96.6 \%$ (Table 4). This justifies the use of AMMI derived measures based on the large numbers of IPCAs results in the most usage of $\mathrm{GxE}$ interaction variations.

Minimum and maximum values of EV1 observed for (G9, G6, G1) and (G7, G5) while corresponding to D1 were (G9, G6, G1) and (G7, G5) absolute values of ASTAB1 for (G9, G6, G1) and (G7, G5) and for SIPC1 were $(\mathrm{G} 7, \mathrm{G} 5, \mathrm{G} 10)$ \& $(\mathrm{G} 11, \mathrm{G} 2)$, of low yield performance (Tables $3 \& 4$ ). 
Table.1 Parentage details of genotypes along with environmental conditions (2017-18)

\begin{tabular}{|c|c|c|c|c|c|c|c|}
\hline Code & Genotype & Parentage & Code & Locations & Latitude & Longitude & $\begin{array}{l}\text { Altitud } \\
\text { e (m) }\end{array}$ \\
\hline G1 & HD2888 & (C 306/T.SPHAEROCOCCUM//HW2004) & E1 & Varanasi & $25^{\circ} 19^{\prime} \mathrm{N}$ & $82^{\circ} 59^{\prime} \mathrm{E}$ & 84 \\
\hline G2 & HI 1612 & (KAUZ//ALTAR84/AOS/3/MILAN/KAUZ/4/HUITES) & E2 & Burdwan & $23^{\circ} 13^{\prime} \mathrm{N}$ & $87^{\circ} 51^{\prime} \mathrm{E}$ & 38 \\
\hline G3 & WH 1235 & (METSO/ER2000/5/2*SERI*3//RL6010/4*YR/3/PASTOR/4/BAV92) & E3 & Coochbehar & $26^{\circ} 34^{\prime} \mathrm{N}$ & $89^{\circ} 44^{\prime} \mathrm{E}$ & 42 \\
\hline G4 & BRW3806 & (NI 5439/MACS 2496) & E4 & Chianki & $24^{\circ} 01^{\prime} \mathrm{N}$ & $84^{\circ} 10 ’ \mathrm{E}$ & 241 \\
\hline G5 & K 1317 & (K0307/K9162) & E5 & Deegh & $26^{\circ} 02^{\prime} \mathrm{N}$ & $80^{\circ} 54^{\prime} \mathrm{E}$ & 121 \\
\hline G6 & DBW2 52 & (PFAU/MILAN/5/CHEN/AE.SQ(TAUS)//BCN/3/VEE\#7/BOW/4/PASTOR) & E6 & Ghaghraghat & $26^{\circ} 54^{\prime} \mathrm{N}$ & $81^{\circ} 56^{\prime} \mathrm{E}$ & 100 \\
\hline G7 & K 8027 & $(\mathrm{HD} 1696 / 2 * \mathrm{~K} 852)$ & E7 & Kalyani & $22^{\circ} 58^{\prime} \mathrm{N}$ & $88^{\circ} 26^{\prime} \mathrm{E}$ & 16 \\
\hline G8 & HD3171 & (PBW343/HD2879 & E8 & Kanpur & $26^{\circ} 26^{\prime} \mathrm{N}$ & $80^{\circ} 19^{\prime} \mathrm{E}$ & 133 \\
\hline \multirow[t]{4}{*}{ G9 } & HI1628 & (FRET2*2/4/SNI/TRAP\#1/3/KAUZ*2/TRAP//KAUZ/5/PFAU/WEAVER//BRAMBLING) & E9 & Purnea & $25^{\circ} 46^{\prime} \mathrm{N}$ & $87^{\circ} 28^{\prime} \mathrm{E}$ & 43 \\
\hline & & & E10 & Pusa & $25^{\circ} 98^{\prime} \mathrm{N}$ & $85^{\circ} 67^{\prime} \mathrm{E}$ & 56 \\
\hline & & & E11 & Ranchi & $23^{\circ} 20^{\prime} \mathrm{N}$ & $85^{\circ} 18^{\prime} \mathrm{E}$ & 644 \\
\hline & & & E12 & Sabour & $25^{\circ} 23^{\prime} \mathrm{N}$ & $87^{\circ} 04^{\prime} \mathrm{E}$ & 42 \\
\hline
\end{tabular}

Table.2 Parentage details of genotypes along with environmental conditions (2018-19)

\begin{tabular}{|c|c|c|c|c|c|c|c|}
\hline Code & Genotype & Parentage & Code & Location & Latitude & Longitude & Altitude \\
\hline G1 & HD 3249 & PBW343*2/KUKUNA//SRTU/3/PBW343*2/KHVAKI & E1 & Kanpur & $26^{\circ} 26^{\prime} \mathrm{N}$ & $80^{\circ} 19^{\prime} \mathrm{E}$ & 126 \\
\hline G2 & HD 2733 & ATTILA/3/TUI/CARC//CHEN/CHTO/4/ATTILA & $\mathrm{E} 2$ & Faizabad & $26^{\circ} 46^{\prime} \mathrm{N}$ & $82^{\circ} 9^{\prime} \mathrm{E}$ & 97 \\
\hline G3 & PBW 781 & $\begin{array}{l}\text { PBW621/4/BW9250*3//Yr10/6* Avocet/3/ BW9250*3//Yr15/6* } \\
\text { Avocet/5/2*PBW } 621\end{array}$ & E3 & Varanasi & $25^{\circ} 19^{\prime} \mathrm{N}$ & $82^{\circ} 59^{\prime} \mathrm{E}$ & 81 \\
\hline G4 & DBW 257 & HUW640/HD3055 & $\mathrm{E} 4$ & Gorakhpur & $26^{\circ} 45^{\prime} \mathrm{N}$ & $83^{\circ} 21^{\prime} \mathrm{E}$ & 84 \\
\hline G5 & DBW 39 & ATTILA/HUI & E5 & IARI-Pusa & $28^{\circ} 38^{\prime} \mathrm{N}$ & $77^{\circ} 09^{\prime} \mathrm{E}$ & 52 \\
\hline G6 & HD 3277 & CHEN/AEG.SQUARROSA//BCN/3/BAV92/4/BERKUT & E6 & Sabour & $25^{\circ} 23^{\prime} \mathrm{N}$ & $87^{\circ} 04^{\prime} \mathrm{E}$ & 46 \\
\hline G7 & RAJ 4529 & PHS 0624/WR1136 & E7 & Purnea & $25^{\circ} 46^{\prime} \mathrm{N}$ & $87^{\circ} 28^{\prime} \mathrm{E}$ & 36 \\
\hline G8 & DBW 187 & NAC/TH.AC//3*PVN/3/MIRLO/BUC/4/2*PASTOR/5/KACHU/6/KACHU & E8 & Banka & $24^{\circ} 53^{\prime} \mathrm{N}$ & $86^{\circ} 55^{\prime} \mathrm{E}$ & 79 \\
\hline G9 & WH 1239 & TAM200/PASTOR//TOBA97 & E9 & RPCAU-Pusa & $25^{\circ} 98^{\prime} \mathrm{N}$ & $25^{\circ} 67 \mathrm{E}$ & 52 \\
\hline G10 & K0307 & K8321/UP2003 & E10 & Ranchi & $23^{\circ} 20^{\prime} \mathrm{N}$ & $85^{\circ} 18^{\prime} \mathrm{E}$ & 651 \\
\hline \multirow[t]{5}{*}{ G11 } & HD 2967 & ALD/CUC//URES/HD2160M/HD2278 & E11 & Chianki & $23^{\circ} 45^{\prime} \mathrm{N}$ & $85^{\circ} 30^{\prime} \mathrm{E}$ & 215 \\
\hline & & & E12 & Dumka & $24^{\circ} 27^{\prime} \mathrm{N}$ & $87^{\circ} 26^{\prime} \mathrm{E}$ & 137 \\
\hline & & & E13 & Kalyani & $22^{\circ} 58^{\prime} \mathrm{N}$ & $88^{\circ} 26^{\prime} \mathrm{E}$ & 11 \\
\hline & & & E14 & Burdhwan & $23^{\circ} 13^{\prime} \mathrm{N}$ & $87^{\circ} 51^{\prime} \mathrm{E}$ & 30 \\
\hline & & & E15 & Shillongani & $26^{\circ} 8^{\prime} \mathrm{N}$ & $91^{\circ} 43^{\prime} \mathrm{E}$ & 86 \\
\hline
\end{tabular}


Table.3 AMMI analysis of genotypes (2017-18)

\begin{tabular}{|c|c|c|c|c|c|c|}
\hline Source & Degree of freedom & MS & Level of significance & $\%$ of Total SS & $\%$ of GxE SS & Cumulative \% SS by PCA's \\
\hline Treatments & 107 & 231.71 & $* * *$ & 89.39 & & \\
\hline Genotypes & 8 & 166.47 & $* * *$ & 4.80 & & \\
\hline Environments & 11 & 1632.53 & $* * *$ & 64.74 & & \\
\hline GxE interaction & 88 & 62.54 & $* * *$ & 19.84 & & \\
\hline IPC1 & 18 & 115.32 & $* * *$ & & 37.72 & 37.72 \\
\hline IPC2 & 16 & 78.09 & $* * *$ & & 22.71 & 60.43 \\
\hline IPC3 & 14 & 52.49 & $* * *$ & & 13.35 & 73.78 \\
\hline IPC4 & 12 & 54.99 & $* * *$ & & 11.99 & 85.77 \\
\hline IPC5 & 10 & 37.82 & $* * *$ & & 6.87 & 92.64 \\
\hline IPC6 & 8 & 30.04 & $* * *$ & & 4.37 & 97.01 \\
\hline IPC7 & 6 & 23.55 & $* * *$ & & 2.57 & 99.58 \\
\hline Residual & 4 & 5.81 & & & & \\
\hline Error & 324 & 9.09 & & & & \\
\hline Total & 431 & 64.35 & & & & \\
\hline
\end{tabular}

Table.4 AMMI analysis of genotypes (2018-19)

\begin{tabular}{|c|c|c|c|c|c|c|}
\hline Source & Degree of freedom & MS & Level of significance & \% of Total SS & \% of GxE SS & Cumulative \% SS by PCA's \\
\hline Treatments & 164 & 226.6779 & $* * *$ & 83.39 & & \\
\hline Genotypes & 10 & 253.3109 & $* * *$ & 5.68 & & \\
\hline Environments & 14 & 1578.168 & $* * *$ & 49.56 & & \\
\hline GxE interaction & 140 & 89.62649 & $* * *$ & 28.15 & & \\
\hline IPC1 & 23 & 190.0607 & $* * *$ & & 34.84 & 34.84 \\
\hline IPC2 & 21 & 132.1767 & $* * *$ & & 22.12 & 56.96 \\
\hline IPC3 & 19 & 91.42374 & $* * *$ & & 13.84 & 70.80 \\
\hline IPC4 & 17 & 74.01184 & $* * *$ & & 10.03 & 80.83 \\
\hline IPC5 & 15 & 55.89603 & $* * *$ & & 6.68 & 87.51 \\
\hline IPC6 & 13 & 55.62916 & $* * *$ & & 5.76 & 93.28 \\
\hline IPC7 & 11 & 38.39033 & $* *$ & & 3.37 & 96.64 \\
\hline Residual & 21 & 20.06844 & & & & \\
\hline Error & 495 & 14.9596 & & & & \\
\hline Total & 659 & 67.64822 & & & & \\
\hline
\end{tabular}


Table.5 Principal components analysis of genotypes (2017-18)

\begin{tabular}{|c|c|c|c|c|c|c|c|c|c|c|c|c|c|c|c|}
\hline & EV1 & EV2 & EV3 & EV5 & EV7 & D1 & D2 & D3 & D5 & D7 & SIPC1 & SIPC2 & SIPC3 & SIPC5 & SIPC7 \\
\hline G1 & 0.1314 & 0.1472 & 0.1210 & 0.0769 & 0.0630 & 6.3572 & 8.6421 & 9.1840 & 9.3368 & 9.4660 & -2.4469 & -0.0460 & -1.4080 & -2.0995 & -3.1828 \\
\hline G2 & 0.0098 & 0.0076 & 0.0170 & 0.0537 & 0.0470 & 1.7340 & 2.0352 & 3.0346 & 5.5861 & 5.8546 & 0.6674 & 0.2305 & -0.7560 & -1.0334 & -0.9600 \\
\hline G3 & 0.1192 & 0.0861 & 0.0610 & 0.0567 & 0.0550 & 6.0540 & 6.9147 & 7.0228 & 7.7961 & 8.0735 & 2.3302 & 3.7004 & 3.1625 & 2.7346 & 4.2003 \\
\hline G4 & 0.0449 & 0.0456 & 0.0336 & 0.0722 & 0.0540 & 3.7155 & 4.8503 & 4.9872 & 7.5772 & 7.6460 & 1.4301 & 2.7087 & 3.2174 & 6.4158 & 5.8597 \\
\hline G5 & 0.0137 & 0.0967 & 0.0866 & 0.0861 & 0.0634 & 2.0562 & 6.4801 & 7.1666 & 8.1295 & 8.1749 & -0.7914 & -3.3117 & -4.6531 & -2.6253 & -2.4446 \\
\hline G6 & 0.0002 & 0.0001 & 0.0224 & 0.0205 & 0.0500 & 0.2365 & 0.2811 & 3.0888 & 3.6766 & 5.3456 & -0.0910 & -0.1533 & 1.1947 & -0.0629 & 1.6934 \\
\hline G7 & 0.1542 & 0.0776 & 0.0792 & 0.0508 & 0.0575 & 6.8857 & 6.9029 & 7.6999 & 7.8331 & 8.2202 & -2.6503 & -2.8500 & -1.3550 & -0.8605 & 0.1483 \\
\hline G8 & 0.0110 & 0.0250 & 0.0281 & 0.0427 & 0.0503 & 1.8356 & 3.4029 & 4.0544 & 5.3494 & 6.0128 & 0.7065 & -0.4687 & -1.4346 & -3.6609 & -4.1156 \\
\hline G9 & 0.0157 & 0.0139 & 0.0510 & 0.0403 & 0.0597 & 2.1967 & 2.7167 & 5.0047 & 5.5894 & 6.4615 & 0.8455 & 0.1900 & 2.0321 & 1.1922 & -1.1988 \\
\hline
\end{tabular}

$\mathrm{EV}=$ Eigenvector, $\mathrm{D}=$ Parameter of Annicchiarico; SIPC1 = SIPC for first IPCA, SIPC 2 = SIPC for first two IPCAs, ..., ASV = AMMI stability value; MASV = Modified AMMI stability value

Table.6 AMMI based estimates of genotypes (2017-18)

\begin{tabular}{|c|c|c|c|c|c|c|c|c|c|c|c|c|c|c|}
\hline & ASTAB1 & ASTAB2 & ASTAB3 & ASTAB5 & ASTAB7 & MASV1 & MASV & ASV1 & ASV & MEAN & GAI & PRVG & MHPRVG & HM \\
\hline G1 & 40.41 & 74.69 & 84.35 & 87.18 & 89.61 & 6.81 & 5.64 & 4.72 & 3.96 & 31.42 & 30.61 & 0.9206 & 0.8979 & 29.72 \\
\hline G2 & 3.01 & 4.14 & 9.21 & 31.20 & 34.28 & 4.87 & 4.16 & 1.19 & 0.96 & 36.52 & 35.53 & 1.0575 & 1.0531 & 34.46 \\
\hline G3 & 36.65 & 47.81 & 49.32 & 60.78 & 65.18 & 5.82 & 4.76 & 4.11 & 3.30 & 34.04 & 33.11 & 0.9908 & 0.9761 & 32.01 \\
\hline G4 & 13.80 & 23.52 & 24.87 & 57.41 & 58.46 & 6.29 & 5.18 & 2.70 & 2.24 & 33.30 & 32.08 & 0.9618 & 0.9414 & 30.66 \\
\hline G5 & 4.23 & 41.99 & 51.36 & 66.09 & 66.83 & 6.54 & 5.57 & 2.84 & 2.72 & 34.66 & 33.35 & 0.9988 & 0.9812 & 31.88 \\
\hline G6 & 0.06 & 0.08 & 9.54 & 13.52 & 28.58 & 4.70 & 4.04 & 0.16 & 0.13 & 36.17 & 35.68 & 1.0623 & 1.0572 & 35.14 \\
\hline G7 & 47.41 & 47.65 & 59.29 & 61.36 & 67.57 & 5.31 & 4.42 & 4.41 & 3.42 & 32.22 & 31.37 & 0.9385 & 0.9250 & 30.44 \\
\hline G8 & 3.37 & 11.58 & 16.44 & 28.62 & 36.15 & 4.96 & 4.23 & 1.66 & 1.49 & 36.74 & 35.82 & 1.0675 & 1.0605 & 34.77 \\
\hline G9 & 4.83 & 7.38 & 25.05 & 31.24 & 41.75 & 4.77 & 4.23 & 1.55 & 1.27 & 34.10 & 33.59 & 1.0021 & 0.9937 & 33.04 \\
\hline
\end{tabular}

ASTAB = AMMI stability; PRVG = Relative performance of genetic values; MHPRVG= (Harmonic mean of relative performance of genetic values;

GAI $=$ Geometric adaptability measure 
Table.7 Principal components analysis of genotypes (2018-19)

\begin{tabular}{|c|c|c|c|c|c|c|c|c|c|c|c|c|c|c|c|}
\hline & EV1 & EV2 & EV3 & EV5 & EV7 & D1 & D2 & D3 & D5 & D7 & SIPC1 & SIPC2 & SIPC3 & SIPC5 & SIPC7 \\
\hline G1 & 0.0068 & 0.0063 & 0.0292 & 0.0440 & 0.0316 & 5.47 & 6.79 & 13.28 & 16.95 & 16.98 & 0.6721 & 1.2277 & -0.5408 & -2.4070 & -2.4964 \\
\hline G2 & 0.0429 & 0.0619 & 0.0604 & 0.0378 & 0.0606 & 13.70 & 20.31 & 22.62 & 22.79 & 26.24 & 1.6843 & 3.7498 & 5.2938 & 5.9807 & 3.2040 \\
\hline G3 & 0.0256 & 0.0387 & 0.0258 & 0.0238 & 0.0500 & 10.58 & 15.99 & 16.00 & 17.52 & 21.17 & 1.3014 & 2.9536 & 3.0151 & 4.4624 & 7.7698 \\
\hline G4 & 0.0180 & 0.0200 & 0.0669 & 0.0465 & 0.0336 & 8.87 & 11.81 & 20.46 & 21.10 & 21.13 & 1.0907 & 2.1660 & -0.4221 & 0.5340 & 0.3238 \\
\hline G5 & 0.0944 & 0.0472 & 0.0525 & 0.0381 & 0.0316 & 20.31 & 20.32 & 22.86 & 23.46 & 23.86 & -2.4984 & -2.5216 & -4.1444 & -3.2038 & -4.3990 \\
\hline G6 & 0.0032 & 0.0141 & 0.0359 & 0.0362 & 0.0527 & 3.76 & 9.14 & 14.89 & 17.60 & 20.36 & 0.4619 & 1.6103 & 3.4301 & 2.4244 & 2.4621 \\
\hline G7 & 0.2130 & 0.1074 & 0.0831 & 0.0735 & 0.0527 & 30.52 & 30.59 & 31.56 & 33.83 & 33.84 & -3.7528 & -3.4548 & -2.2543 & 999 & 0.1576 \\
\hline G8 & 0.0154 & 0.0873 & 0.0587 & 0.0379 & 0.0461 & 8.20 & 22.57 & 22.63 & 22.97 & 24.21 & 1.0088 & -1.8875 & -2.1491 & -1.2515 & -2.4167 \\
\hline G9 & 0.0016 & 0.0029 & 0.0050 & 0.0553 & 0.0396 & 2.62 & 4.33 & 5.88 & 15.98 & 16.00 & -0.3228 & -0.7966 & -0.1801 & -3.2776 & -3.4798 \\
\hline G10 & 0.0309 & 0.0199 & 0.0134 & 0.0592 & 0.0542 & 11.62 & 12.63 & 12.66 & 21.90 & 22.69 & -1.4292 & -2.1115 & -1.9940 & -4.5138 & -3.3179 \\
\hline G11 & 0.0481 & 0.0943 & 0.0690 & 0.0478 & 0.0473 & 14.51 & 24.50 & 25.15 & 25.78 & 26.57 & 1.7841 & -0.9353 & -0.0542 & 1.3620 & 2.1925 \\
\hline
\end{tabular}

Table.8 AMMI based estimates of genotypes (2018-19)

\begin{tabular}{|c|c|c|c|c|c|c|c|c|c|c|c|c|c|c|}
\hline & ASTAB1 & ASTAB2 & ASTAB3 & ASTAB5 & ASTAB7 & ASV & ASV1 & MASV & MASV1 & MEAN & GAI & HM & PRVG & MHPRVG \\
\hline G1 & 3.67 & 5.91 & 26.10 & 46.70 & 46.86 & 1.0100 & 1.1954 & 4.1596 & 4.5159 & 48.81 & 48.49 & 48.16 & 1.0358 & 1.0300 \\
\hline G2 & 23.07 & 54.03 & 69.42 & 70.78 & 103.42 & .9553 & .3619 & 2246 & 7.3708 & 48.16 & 47.53 & 46.86 & .0170 & 1.0076 \\
\hline G3 & 13.77 & 33.59 & 33.61 & 42.19 & 70.46 & 2.3232 & 2.6326 & 5.0702 & 5.9705 & 45.93 & 45.34 & 44.76 & 0.9690 & 0.9625 \\
\hline G4 & 9.67 & 18.07 & 61.31 & 66.25 & 66.52 & 1.7406 & 2.0265 & 4.7818 & 5.3608 & 45.33 & 44.88 & 44.40 & 0.9602 & 0.9512 \\
\hline G5 & 50.75 & 50.76 & 67.76 & 72.92 & 76.74 & 3.1354 & 3.9347 & 4.4604 & 5.2721 & 45.79 & 45.34 & 44.88 & 0.9710 & 0.9597 \\
\hline G6 & 1.73 & 11.31 & 32.69 & 47.63 & 69.04 & 1.2864 & 1.3594 & 5.1141 & 5.8188 & 47.06 & 46.54 & 45.98 & 0.9945 & 0.9881 \\
\hline G7 & 114.52 & 115.16 & 124.47 & 149.40 & 149.59 & 4.7190 & 5.9178 & 6.0329 & 7.2914 & 45.67 & 44.85 & 44.04 & 0.9646 & 0.9455 \\
\hline G8 & 8.27 & 69.16 & 69.60 & 72.26 & 84.98 & 3.1608 & 3.3034 & 5.2706 & 6.1060 & 51.17 & 50.61 & 50.07 & 1.0814 & 1.0747 \\
\hline G9 & 0.85 & 2.48 & 4.93 & 45.90 & 46.01 & 0.6234 & 0.6950 & 4.2612 & 4.4906 & 50.41 & 49.95 & 49.46 & 1.0665 & 1.0615 \\
\hline G10 & 16.61 & 19.99 & 20.08 & 73.80 & 81.54 & 1.9190 & 2.3520 & 5.3592 & 6.1410 & 45.50 & 44.97 & 44.45 & 0.9615 & 0.9543 \\
\hline G11 & 25.88 & 79.56 & 84.57 & 90.24 & 99.22 & 3.5225 & 3.9102 & 5.5448 & 6.4681 & 46.71 & 45.65 & 44.51 & 0.9785 & 0.9660 \\
\hline
\end{tabular}




\section{Int.J.Curr.Microbiol.App.Sci (2020) 9(5): 1257-1270}

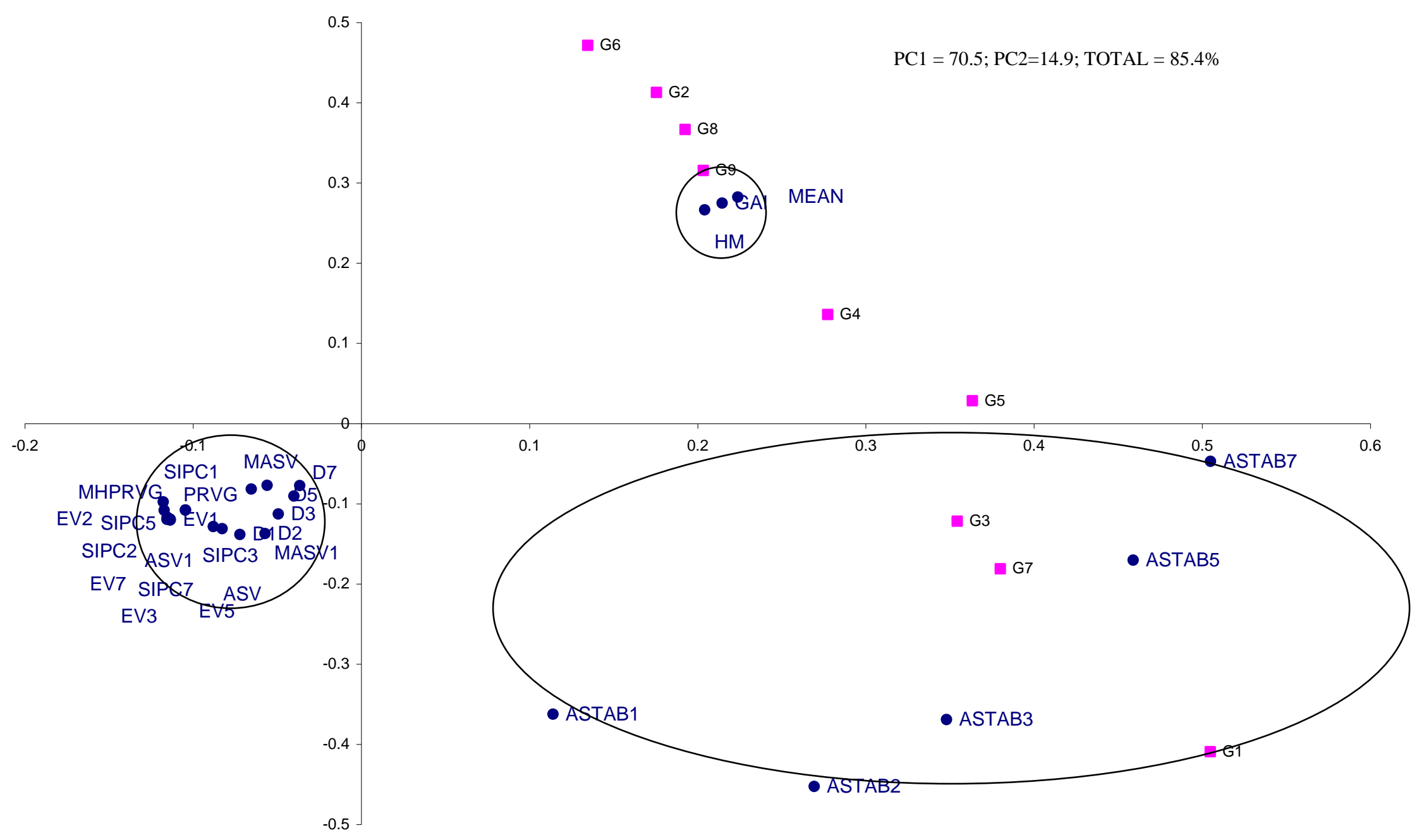

Figure.1 Biplot analysis of genotypes and AMMI based estimates (2017-18) 


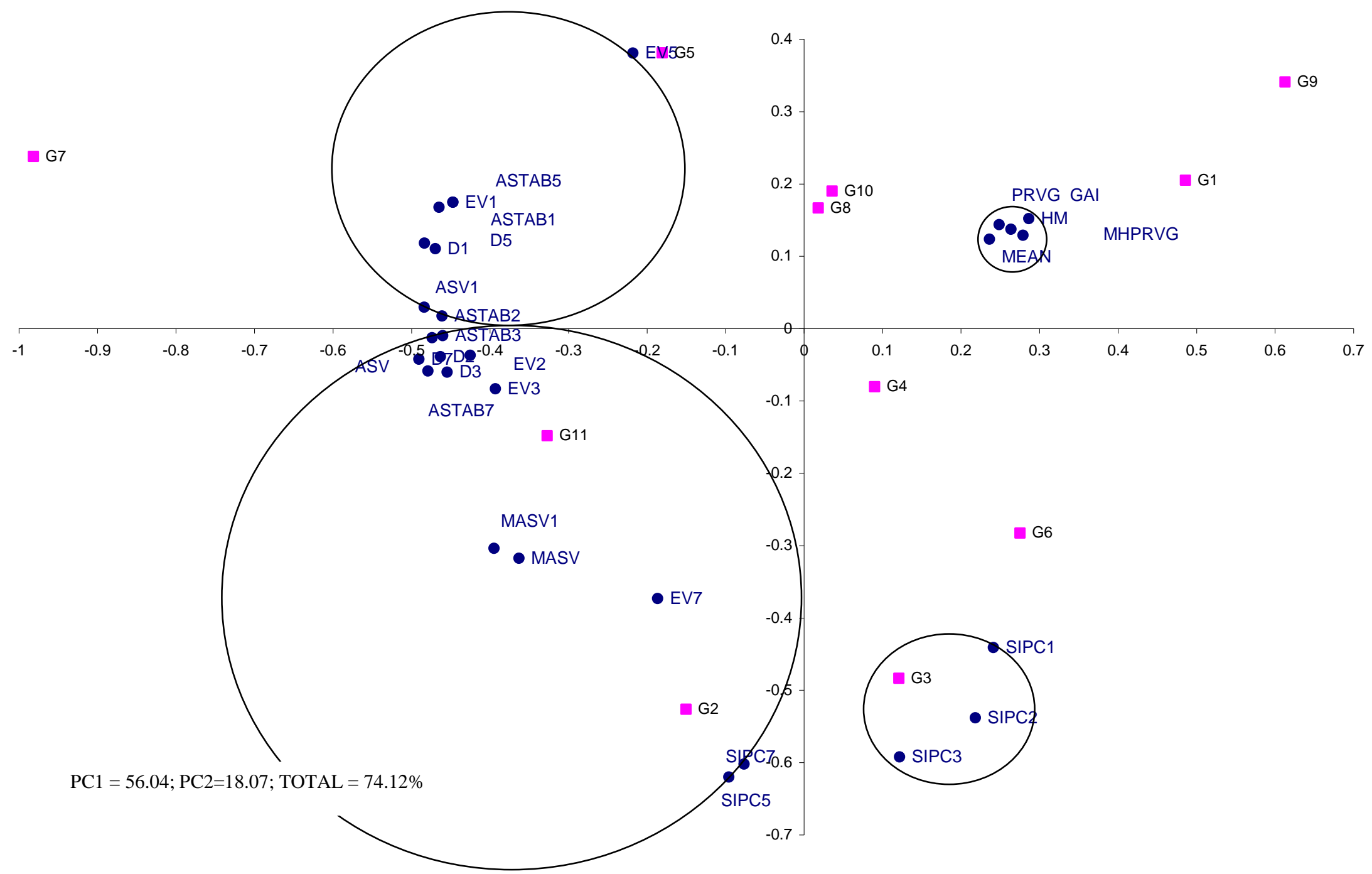

Figure.2 Biplot analysis of genotypes and AMMI based estimates (2018-19) 
Genotypes EV2 pointed towards (G9, G1, G6) as desirable at the same time undesirable genotypes (G7, G11), for values of D2 genotypes were $(\mathrm{G} 9, \mathrm{G} 1, \mathrm{G} 6)$ \& $(\mathrm{G} 7, \mathrm{G} 11)$, whereas as per criterion of SIPC2 were (G7, $\mathrm{G} 5, \mathrm{G} 10)$ \& (G2, G3) and of ASTAB2 were (G9, G1, G6) \& (G7, G11) (Tables 4 and 5). In recent studies, agronomic concept of stability would be more preferred instead of static concept of stability (Karimizadeh et al., 2016).

Using first two IPCAs in stability analysis could benefits dynamic concept of stability in identification of the stable high yielder genotypes. ASV and ASV1 recommended (G9, G1, G6) as of stable performance and unsuitable were G7, G11 as well as G7, G5 by measures respectively (Table 8). First two IPCAs in ASV and ASV1 measures used $56.9 \%$ of $\mathrm{GxE}$ interaction. Minimum values EV3 preferred G9, G10, G3 as well of unstable performance of G7, G11 while SIPC3 pointed towards G5, G7, G8 and G2, G6 whereas D3 for G9, G10, G1 \& G7, G11; ASTAB3 measure considered G9, G10, G1 \& G7, G11 (Table 4).

G3, G6, G2 preferred by least values EV5 and maximum values found for G7, G10, measure SIPC5 identified G10, G9, G5 and G2, G3 whereas D5 considered G9, G2, G3, as suitable \& G7, G11 as unsuitable ones; ASTAB5 selected G3, G9, G1 as suitable \& G7, G11 as unsuitable genotypes. According to D7 minimum values G9, G1, G6 were genotypes of stable yield while G7 and G11 as undesirable; SIPC7 observed G5, G9, G10, as of stable \& G3, G2 of unstable yield (Tables 4 and 5). EV7 pointed towards G1, G5, G4 \& G2, G10.

Measure ASTAB7 identified G9, G1, G4 as desirable and G7, G9 for unstable behavior over the studied environments. Composite measure MASV selected G9, G5, G1 as of stable performance and G2, G7 not recommended for cultivation due to unstable yield behavior. Moreover, similar performance cited by MASV1 as G9, G1, G5 for desirable and G2, G7 vice versa. Genotypes G8 G9 and G1 along with G4 G10 by Mean, G7, G4 by GAI and HM, G4, G10 by PRVG and G7, G4 by MHPRVG measures based on yield of genotypes across environments of study.

\section{Biplot analysis}

AMMI based measures had distributed among four quadrants in biplot analysis based on first two significant principal components. AMMI based measures along with yield could be divided into four clusters of measures were observed in Figure 1.

Largest group I clubbed measures as MASV1, ASV, MASV, D2, D3, D7, with EV2, EV3, SIPC5, SIPC7, EV7. Group II contains ASTAB1, ASTAB2, ASTAB5, D1, D5, EV1, EV5, ASV1 whereas yield based measures exhibited close proximity and placed close to each other as in separate group and SIPC1, SIPC2, SIPC3 measures in last group.

AMMI a based measure relates to different concepts of yield stability and would be useful to wheat researchers attempt to identify and recommend genotypes with high, stable and predictable yield across environments (Shahriari et al., 2018). Clustering of genotypes average yield along with others mean based measures observed with SIPC measures.

\section{Acknowledgements}

The wheat genotypes were evaluated at coordinated centers of AICW\&BIP across the country. Authors sincerely acknowledge the hard work of all the staff for field evaluation and data recording. 


\section{References}

Agahi K., Jafar Ahmadi, Hassan Amiri Oghan, Mohammad Hossein Fotokian and Sedigheh Fabriki Orang (2020) Analysis of genotype $\times$ environment interaction for seed yield in spring oilseed rape using the AMMI model. Crop Breeding and Applied Biotechnology 20(1): e26502012

Annicchiarico, P., 1997. Joint regression vs AMMI analysis of genotypexenvironment interactions for cereals in Italy. Euphytica 94: 53-62.

Ajay B. C., J. Aravind, R. Abdul Fiyaz, Narendra Kumar, Chuni Lal, K. Gangadhar, Praveen Kona, M. C. Dagla and S. K. Bera 2019 Rectification of modified AMMI stability value (MASV) Indian J. Genet., 79(4): 726731

Bocianowski, J., Niemann, J., Nowosad, K., 2019a. Genotype-by-environment interaction for seed quality traits in interspecific cross-derived Brassica lines using additive main effects and multiplicative interaction model. Euphytica 215:7.

Gauch HG (2013) A Simple Protocol for AMMI Analysis of Yield Trials. Crop Science 53:1860-1869.

Guilly, S., Thomas, D., Audrey, T., Laurent, B., Jean-Yves, H., 2017. Analysis of multi environment trials (MET) in the sugarcane breeding program of Re'union Island. Euphytica 213, 213.

Kamila, N., Alina, L., Wiesława, P., Jan, B.,2016. Genotype by environment interaction for seed yield in rapeseed (Brassica napus L.) using additive main effects and multiplicative interaction model. Euphytica 208: 187-194.

Kendal, E., Tekdal, S., 2016. Application of AMMI Model for Evolution Spring Barley Genotypes in MultiEnvironment Trials. Bangladesh Journal of Botany45(3), 613-620.

Mohammadi R and Amri A(2008) Comparison of parametric and nonparametric methods for selecting stable and adapted durum wheat genotypes in variable environments. Euphytica 159: 419-432.

Mohammadi, M., Sharifi,P., Karimizadeh,R., Jafarby,J.A., Khanzadeh,H., Hosseinpour, T., Poursiabidi,M.M., Roustaii,M., Hassanpour Hosni,M., Mohammadi, P., 2015. Stability of grain yield of durum wheat genotypes by AMMI model. Agriculture \& Forestry 61(3): 181-193.

Ndhlela,T.,Herselman,L.,Magorokosho,C.,Set imela,P.,Mutimaamba,C.,Labuschagne M., 2014. Genotype $\times$ environment Interaction of Maize Grain Yield Using AMMI Biplots. Crop Science 54: 19921999.

Nowosad, K., Liersch, A., Popławska, W., Bocianowski, J.,2016. Genotype by environment interaction for seed yield in rapeseed (Brassica napus L.) using additive main effects and multiplicative interaction model. Euphytica 208:187194

Nowosad, K., Tratwal, A., Bocianowski, J.,2018. Genotype by environment interaction for grain yield in spring barley using additive main effects and multiplicative interaction model. Cereal Research Communications 46(4):729_ 738.

Purchase, J.L., 1997. Parametric analysis to describe $\mathrm{G} \times \mathrm{E}$ interaction and yield stability in winter wheat. Ph.D. thesis. Dep. of Agronomy, Faculty of Agriculture, Univ. of the Orange Free State, Bloemfontein, South Africa.

Rao, A.R., Prabhakaran, V.T., 2005. Use of AMMI in simultaneous selection of genotypes for yield and stability. Journal of the Indian Society of Agricultural Statistics 59: 76-82. 
Resende MDV de and Duarte JB. 2007. Precision and quality control in variety trials. Pesquisa Agropecuaria Tropical. 37(3): 182-194.

Shahriari Z, Heidari B, Dadkhodaie A ., 2018. Dissection of genotype $\times$ environment interactions for mucilage and seed yield in Plantago species: Application of AMMI and GGE biplot analyses. PLoS ONE 13(5): e0196095

Sneller, C.H., Kilgore-Norquest, L., Dombek,D., 1997. Repeatability of yield stability statistics in soybean. Crop Science 37: 383-390.

Tekdal, S., Kendal, E., 2018. AMMI Model to Assess Durum Wheat Genotypes in Multi-Environment Trials, Journal of Agricultural Science and Technology 20, 153-166.

Tena, E., Goshu, F., Mohamad, H., Tesfa, M.,
Tesfaye, D., Seife, A.,2019. Genotypexenvironment interaction by AMMI and GGE-biplot analysis for sugar yield in three crop cycles of sugarcane (Saccharumofficinirum L.) clones in Ethiopia. Cogent Food \& Agriculture 5: 1-14.

Zali H, Farshadfar E, Sabaghpour SH, Karimizadeh R (2012) Evaluation of genotype $\times$ environment interaction in chickpea using measures of stability from AMMI model. Annals of Biological Research 3(7): 3126-3136.

Zobel, R., 1994. Stress resistance and root systems. In Proceedings of the Workshop on Adaptation of Plants to Serious Stresses. 1-4 August. INTSORMIL Publication 94-2, Institute of Agriculture and Natural Recourses. Lincoln, USA: University of Nebraska.

\section{How to cite this article:}

Ajay Verma and Singh. G. P. 2020. Wheat Genotypes Evaluated under North Eastern Plains Zone of the Country for Genotype $\mathrm{X}$ Environment Interaction Analysis. Int.J.Curr.Microbiol.App.Sci. 9(05): 1251-1270. doi: https://doi.org/10.20546/ijcmas.2020.905.140 